\title{
Bacteriemia relacionada con catéter permanente por Chryseobacterium indologenes en un paciente pediátrico con leucemia linfoblástica aguda
}

\section{Chryseobacterium indologenes indwelling catheter-related bacteremia in a pediatric patient with acute lymphoblastic leukemia}

Irina Jeanette Bercholc-Urinowsky, ${ }^{1}$ Rodrigo Ugalde-Resano, ${ }^{2}$ Jorge Monge-Urrea ${ }^{3}$

\begin{abstract}
Resumen
INTRODUCCIÓN: Chryseobacterium indologenes es un microorganismo oportunista, bacilo gramnegativo, responsable de bacteriemias en pacientes inmunocomprometidos y en quienes utilizan dispositivos intravasculares. Su incidencia se ha incrementado como consecuencia de la cada vez mayor prescripción de antibióticos de amplio espectro. CASO CLínICO: Niño de tres años, con leucemia linfoblástica aguda, en quien se colocó un dispositivo Port-a-Cath tres meses luego del diagnóstico inicial. Se hospitalizó 4 meses después para recibir un esquema de quimioterapia; acudió a su domicilio sin eventualidades y presentó reporte normal de biometría hemtática completa. Al permeabilizar la vía tuvo datos de bacteriemia; se sospechó infección relacionada con el catéter, se hemocultivó por vía central y periférica y se inició tratamiento con meropenem $(40 \mathrm{mg} / \mathrm{kg} /$ dosis) y vancomicina $(10 \mathrm{mg} / \mathrm{kg} /$ dosis), y se retiró el catéter permanente. En ambos hemocultivos hubo crecimiento de Chryseobacterium indologenes. Recibió 12 días de antibiótico y, posteriormente, se cambió a levofloxacina, por resultado de antibiograma por siete días más. Tuvo mejoría en las 48 horas siguientes al inicio de los antibióticos y retiro del catéter puerto sin complicaciones. Los hemocultivos posteriores se reportaron negativos y continuó el esquema de quimioterapia.

CONCLUSIÓN: C. indologenes es un problema de salud pública emergente debido a la gran resistencia a los antibióticos de indicación empírica en bacteriemias por gramnegativos. El tratamiento aún no está estandarizado pero puede ser exitoso luego del retiro permanente del dispositivo, o intentar rescatar el dispositivo mediante tratamiento antimicrobiano, de acuerdo con las pruebas de susceptibilidad.
\end{abstract}

PALABRAS CLAVE: Chryseobacterium indologenes; bacilos gramnegativos; Port-a-Cath; leucemia linfoblástica; meropenem; vancomicina; levofloxacina.

\section{Abstract}

BACKGROUND: Chryseobacterium indologenes is an opportunistic gram-negative bacillus responsible for bloodstream infections in immunocompromised patients and in those with indwelling intravascular devices. Recently, its incidence has increased due to a rise in the use of broad-spectrum antibiotics.

CLINICAL CASE: A 3-year-old male patient with acute lymphoblastic leukemia underwent Port-a-Cath placement three months after initial diagnosis. He was hospitalized to receive chemotherapy, without eventualities at home and a normal cellular blood count. When the indwelling catheter was accessed, the patient presented signs of bacteremia, and a catheter-related bacteremia was suspected. Central and peripheral blood cultures were obtained, and meropenem (40mg/kg/dose) and vancomycin (10mg/ $\mathrm{kg} /$ dose) were started, and the permanent catheter was removed. Blood cultures grew Chryseobacterium indologenes from both central and peripheral line specimens. He received a twelve-day course of IV meropenem and vancomycin, and later switched
${ }^{1}$ Residente de tercer año de Pediatría, Hospital Central Sur de Alta Especialidad de Petróleos Mexicanos, Ciudad de México.

${ }^{2}$ Residente de segundo año de Cirugía general, Hospital Central Norte de Petróleos Mexicanos, Ciudad de México. ${ }^{3}$ Residente de Hematología y Oncología médica, Weill Cornell Medicine, Nueva York, EUA.

Recibido: 28 de julio 2017

Aceptado: 26 de marzo 2018

\section{Correspondencia}

Irina Jeanette Bercholc Urinowsky ibercholc@gmail.com

Este artículo debe citarse como Bercholc-Urinowsky IJ, Ugalde-Resano R, Monge-Urrea J. Bacteriemia relacionada con catéter permanente por Chryseobacterium indologenes en un paciente pediátrico con leucemia linfoblástica aguda. Acta Pediatr Mex. 2018;39(5):283-288. 
to levofloxacin as per antimicrobial susceptibility testing and completed a seven-day course. He markedly improved within 48 hours of starting antibiotics and permanent catheter removal, no further complications. Surveillance blood cultures were negative. He was able to complete his chemotherapy regimen.

CONCLUSSION: C. indologenes is an emerging public health problem due to its frequent resistance to antibiotics routinely used for the empiric treatment of gram-negative bacteremia. The treatment, not yet standardized, can be successful by prompt the removal of indwelling catheters, or try to rescue the device by antimicrobial therapy carefully tailored to susceptibility testing.

KEYWORDS: Chryseobacterium indologenes; Gram negative bacilli; Port-a-Cath; Lymphoblastic leukemia; Meropenem; Vancomycin; Levofloxacin.

\section{INTRODUCCIÓN}

Chryseobacterium indologenes, también conocido como Flavobacterium indologenes, forma parte del grupo de bacilos gramnegativos no fermentadores, inmóvil, catalasa y oxidasa positivas, indol positivo. ${ }^{1,2}$ Su hallazgo en humanos es excepcional aunque se encuentra ampliamente distribuido en la naturaleza. Sobre todo, causa infecciones en pacientes con hospitalizaciones prolongadas y aplicación a largo plazo de dispositivos permanentes y exposición extendida a antibióticos de amplio espectro. ${ }^{2,3}$

En los últimos años se ha incrementado su incidencia como agente causal de bacteriemias, neumonías, infecciones asociadas con dispositivos permanentes, peritonitis e infecciones de vías urinarias, entre otras. ${ }^{1,2}$ Debido a la prescripción libre y empírica de antibióticos $C$. indologenes ha surgido como un problema de salud importante, por su gran resistencia antibacteriana y la capacidad de producir betalactamasas de espectro extendido y metalo betalactamasas. ${ }^{1}$

En 2015 se publicó en México el primer caso de sepsis grave secundaria a bacteriemia por $C$. indologenes en un paciente de 59 años. Éste es el segundo caso que se reporta en nuestro país y el primero en un paciente pediátrico. ${ }^{4}$

\section{CASO CLÍNICO}

Paciente de tres años, con diagnóstico de leucemia linfoblástica aguda L2 Pre B Calla negativo, de riesgo bajo por edad y carga tumoral, que se encuentra en remisión completa continua en su cuarto mes. Tres meses después del diagnóstico inicial se le colocó un dispositivo Port-a-Cath. Al cuarto mes se hospitalizó para recibir el siguiente ciclo de quimioterapia de consolidación, con esquema CAP-VAP (6-mercaptopurina, ciclofosamida, citarabina, vincristina). Durante la permanencia en su domicilio no hubo eventualidades y la biometría hemática completa solo registró elevación de la cuenta plaquetaria. (Cuadro 1) Al permeabilizar la vía, el paciente tuvo fiebre, escalofríos, cefalea y dolor abdominal pero permaneció hemodinámicamente estable. Debido a que se sospechó infección relacionada con el catéter se tomó un hemocultivo central y periférico y se inició tratamiento con meropenem (40 mg/kg/dosis) y vancomicina (10 mg/kg/dosis) y se retiró el catéter permanente.

En ambos hemocultivos hubo crecimiento de $C$. indologenes. Al término de 12 días de esquema intravenoso con meropenem y vancomicina se modificó a levofloxacina por mostrar susceptibilidad en el antibiograma (Cuadro 2) y se continuó durante siete días más. 
Cuadro 1. Biometría hemática al ingreso

\begin{tabular}{|l|c|c|}
\hline Estudio & Referencia & Valor \\
\hline Leucocitos $\left(\times 10^{3} / \mu \mathrm{L}\right)$ & $8.5(5-15.5)$ & 4.6 \\
\hline Neutrófilos $\left(\times 10^{3} / \mu \mathrm{L}\right)$ & $4.4(1.5-8.5)$ & 2.11 \\
\hline Linfocitos $\left(\times 10^{3} / \mu \mathrm{L}\right)$ & $3.1(1.5-6.8)$ & 1.44 \\
\hline Monocitos $\left(\mathrm{x} 10^{3} / \mu \mathrm{L}\right)$ & 0.4 & 14.32 \\
\hline Eritrocitos $\left(\times 10^{6} / \mu \mathrm{L}\right)$ & $4.1-5.10$ & 5.27 \\
\hline Hemoglobina $(\mathrm{g} / \mathrm{dL})$ & $12.5(11.5)$ & 12.6 \\
\hline Hematocrito $(\%)$ & $37(34)$ & 43.1 \\
\hline VMC $(\mathrm{fL})$ & $90(78)$ & 81.8 \\
\hline HCM $(\mathrm{pg} / \mathrm{eritrocito})$ & $34(31.0)$ & 23.9 \\
\hline Plaquetas $\left(\mathrm{x} 10^{3} / \mu \mathrm{L}\right)$ & $150-350$ & 645
\end{tabular}

VMC: volumen corpuscular medio. HCM: hemoglobina corpuscular media. Referencias tomadas y adaptadas de: Engorn B, Flerlage J. Table 14-1: Age-Specific Blood Cell Indices y Table 14-6: Age-Specific Leukocyte Differential de The Harriet Lane Handbook, Twentieth Edition, Elsevier, 2014.

Cuadro 2. Antibiograma de Chryseobacterium indologenes

\begin{tabular}{|l|l|}
\hline Amikacina & $\mathrm{S}$ \\
\hline Ciprofloxacina & $\mathrm{S}$ \\
\hline Gentamicina & $\mathrm{S}$ \\
\hline Levofloxacina & $\mathrm{S}$ \\
\hline Imipenem & $\mathrm{S}$ \\
\hline Meropenem & $\mathrm{S}$ \\
\hline Tobramicina & $\mathrm{S}$ \\
\hline Piperacilina/Tazobactam & $\mathrm{S}$ \\
\hline Aztreonam & $\mathrm{R}$ \\
\hline Ceftazidima & $\mathrm{R}$ \\
\hline Cefepima & $\mathrm{R}$ \\
\hline Ceftriaxona & $\mathrm{R}$ \\
\hline Cefotaxima & $\mathrm{R}$ \\
\hline Ticarcilina/clavulanato & $\mathrm{R}$ \\
\hline Trimetoprima/sulfametoxazol & $\mathrm{R}$ \\
\hline
\end{tabular}

S: susceptible; R: resistente.
Luego de 48 horas del inicio de los antibióticos hubo mejoría, sin complicaciones. Los hemocultivos de control resultaron negativos y pudo completar su esquema de quimioterapia.

\section{DISCUSIÓN}

C. indologenes es una bacteria que se encuentra en superficies húmedas y fuentes de agua del medio hospitalario, como: catéteres vasculares permanentes, tubos de alimentación y humidificadores. ${ }^{1,2}$ Bonten y su grupo, en 1993, fueron los primeros en aislar una cepa de C. indologenes de un aspirado traqueal de un paciente con neumonía asociada con el ventilador. ${ }^{2}$ En 1996, Hsueh y colaboradores publicaron, por primera vez, la relación entre infección por $C$. indologenes y la permanencia de dispositivos. ${ }^{2,5}$

El programa de vigilancia antimicrobiana SENTRY se creó en enero de 1997 y consiste en una red mundial de hospitales centinela y laboratorios que recopilan resultados de los patógenos que causan infecciones adquiridas en la comunidad y nosocomiales, para poder seguir sus patrones de sensibilidad y resistencia antimicrobiana. ${ }^{6}$ En Latinoamérica participaron 12 ciudades de siete países, México fue uno de ellos, con tres centros hospitalarios en la Ciudad de México de 1997 a $2002 .^{6}$

En Latinoamérica se reportó una frecuencia de Chryseobacterium species de $6.8 \%$ del total de aislamientos de bacilos gramnegativos no fermentadores. ${ }^{7}$ La incidencia mundial reportada fue de $0.27 \%$ de bacilos gramnegativos no fermentadores y $0.03 \%$ de todos los aislados de bacterias. ${ }^{8-10}$ En 2013, Chen y colaboradores analizaron 215 muestras de pacientes infectados por $C$. indologenes y describieron la correlación entre esta bacteria y el tratamiento con colistina y tigeciclina, y que al incrementar el consumo de estos antibióticos, aumentó su incidencia. ${ }^{2}$ 
Aún se conoce poco de los factores de virulencia de las especies Chryseobacterium, pero dentro de las características que posee, la producción de biofilm sobre dispositivos permanentes y la actividad proteasa pueden participar en el inicio de bacteriemia o complicaciones más graves. ${ }^{2,3,5}$

Con los cultivos y antibiogramas se ha podido observar que en la mayoría de los casos reportados el tratamiento antibiótico de elección son las nuevas quinolonas (garenoxacina, getifloxacina, levofloxacina) porque tienen una susceptibilidad mayor a 95\%, seguidas de trimetoprima-sulfametoxazol con una suceptibilidad de 95\%, piperacilina-tazobactam con 90\% de susceptibilidad; y con $85 \%$ de susceptibilidad criprofloxacina, cefepime, ceftazidima, piperacilina y rifampicina..$^{8,11}$

Hasta ahora no se dispone de un consenso del tratamiento ideal; existen conductas diversas y opuestas. Lin y su grupo ${ }^{11,12}$ sugieren el retiro inmediato de los dispositivos intravenosos para el tratamiento oportuno, debido a la amplia resistencia antimcrobiana y la dificultad de pe- netración de los medicamentos a los dispositivos recubiertos con biofilm. Hay autores que sugieren que en algunos casos los dispositivos pueden rescatarse administrando terapia lock, que consiste en aportar una alta concentración de antibiótico y dejarlo encerrado dentro del dispositivo. ${ }^{13}$ Chen y colaboradores sugieren la permanencia de dispositivos intravenosos en pacientes seleccionados, siempre resaltando la importancia de indicar el tratamiento conforme a la sensibilidad y tiempo apropiado; con esto disminuye la mortalidad incluso en $50 \%$ con respecto a pacientes $\sin$ tratamiento adecuado. ${ }^{2,12}$

En el Cuadro 3 se describe la demografía y características clínicas de infección por $C$. indologenes en los pacientes pediátricos con enfermedades oncológicas e infección asociada con dispositivos intravenosos permanentes actualmente reportados en la bibliografía, incluido el nuestro. ${ }^{4,14,15} \mathrm{En}$ los dos casos reportados por Husueh ${ }^{4,11}$ pudo rescatarse el dispositivo mediante tratamiento médico, mientras que los otros dos, incluido éste, a pesar de haber recibido tratamiento antibiótico, se decidió el retiro del catéter permanente antes de las 48 horas, con una adecuada recuperación.

Cuadro 3. Demografía y características clínicas de la infección por C. indologenes en pacientes pediátricos con enfermedades oncológicas e infección

\begin{tabular}{|c|c|c|c|c|c|c|c|c|}
\hline$n$ & $\begin{array}{c}\text { Autor y } \\
\text { año de } \\
\text { publicación }\end{array}$ & Edad & Género & Comorbilidad & $\begin{array}{l}\text { Dispositivo } \\
\text { médico }\end{array}$ & $\begin{array}{l}\text { Síndrome } \\
\text { clínico }\end{array}$ & Antibiótico & Resultado \\
\hline 1 & $\begin{array}{c}\text { Hsueh et al, } \\
1996^{4,11}\end{array}$ & $\begin{array}{c}5 \\
\text { meses }\end{array}$ & Femenino & $\begin{array}{l}\text { Neuroblastoma, } \\
\text { quimioterapia }\end{array}$ & $\begin{array}{l}\text { Catéter } \\
\text { Hickman }\end{array}$ & bacteriemia & $\begin{array}{c}\text { No } \\
\text { especificado }\end{array}$ & $\begin{array}{l}\text { Recuperación } \\
\text { sin retiro de } \\
\text { catéter (después } \\
\text { de } 3 \text { días) }\end{array}$ \\
\hline 2 & $\begin{array}{c}\text { Hsueh et al, } \\
19964,11\end{array}$ & $\begin{array}{c}1 \\
\text { mes }\end{array}$ & Femenino & $\begin{array}{l}\text { Hepatoblastoma, } \\
\text { quimioterapia }\end{array}$ & Port-A-Cath & bacteriemia & $\begin{array}{c}\text { No } \\
\text { especificado }\end{array}$ & $\begin{array}{l}\text { Recuperación } \\
\text { sin retiro de } \\
\text { catéter (después } \\
\text { de } 3 \text { días) }\end{array}$ \\
\hline 3 & $\begin{array}{l}\text { Kodama et } \\
\text { al, } 2013^{14}\end{array}$ & $\begin{array}{c}3 \\
\text { años }\end{array}$ & Femenino & $\begin{array}{l}\text { Leucemia mieloide } \\
\text { aguda, transplante } \\
\text { de células madre } \\
\text { no relacionado }\end{array}$ & $\begin{array}{l}\text { Catéter } \\
\text { venoso } \\
\text { central }\end{array}$ & bacteriemia & $\begin{array}{l}\text { Ciprofloxacina } \\
\text { y minociclina }\end{array}$ & $\begin{array}{l}\text { Recuperación } \\
\text { con retiro de } \\
\text { catéter }\end{array}$ \\
\hline 4 & $\begin{array}{l}\text { Reporte de } \\
\text { caso }\end{array}$ & $\begin{array}{c}3 \\
\text { años }\end{array}$ & Masculino & $\begin{array}{l}\text { Leucemia linfo- } \\
\text { blástica aguda, } \\
\text { quimioterapia }\end{array}$ & Port-A-Cath & bacteriemia & $\begin{array}{l}\text { Meropenem, } \\
\text { vancomicina, } \\
\text { levofloxacino }\end{array}$ & $\begin{array}{l}\text { Recuperación } \\
\text { con retiro de } \\
\text { catéter }\end{array}$ \\
\hline
\end{tabular}


En 2016 Aykac y colaboradores publicaron la recopilación de seis casos en pacientes pediátricos de 2012 a 2015, donde uno de sus pacientes tuvo bacteriemia relacionada con el catéter, que al no contar con otra vía de acceso se decidió no remover el dispositivo; a pesar del tratamiento antibiótico al que era sensible el paciente falleció. Por eso los autores sugieren que si un catéter está infectado por C. indologenes debe retirarse cuanto antes. ${ }^{16}$

En el caso de nuestro paciente se indicó fluoroquinolonas debido a la susceptibilidad en el antibiograma y por ser el fármaco de primera línea, como lo comentan Kirby y su grupo. ${ }^{8}$ Jackson y colaboradores reportaron una serie de estudios donde se demuestra la seguridad de levofloxacina en pacientes pediátricos con muy bajo índice de complicaciones, por lo que está indicado en casos sensibles al medicamento. ${ }^{17}$

La información con respecto al tratamiento adecuado es limitada. Ambas conductas de tratamiento son válidas pero es importante individualizar cada caso y valorar la necesidad de conservar los catéteres permanentes.

\section{CONCLUSIONES}

La infección por $C$. indologenes es un problema de salud pública emergente debido a la gran resistencia a los antibióticos indicados de manera empírica en bacteriemias por gramnegativos; esto ha conferido la habilidad para producir betalactamasas de espectro extendido y metalo betalactamasas. Aún no se cuenta con un protocolo de tratamiento estandarizado, pero este deberá ir encaminado a la necesidad de conservar los accesos vasculares y la seguridad del paciente.

\section{REFERENCIAS}

1. Malini A, et al. Nonfermenting gram-negative bacilli infections in a tertiary care hospital in Kolar, Karnataka. J Lab Physicians. 2009;1(2):62-66. doi: 10.4103/0974-2727.59701
2. Chen F, et al. Clinical and epidemiological features of Chryseobacterium indologenes infections: Analysis of 215 cases. J Microbiol Immunol Infec. 2013;46(6):425-32. https://doi. org/10.1016/j.jmii.2012.08.007

3. Chang $\mathrm{Y}$, et al. Identification, epidemiological relatedness, and biofilm formation of clinical Chryseobacterium indologenes isolates from central Taiwan. J Microbiol Immunol Infec. 2015;48(5):559-64. DOI: https://doi.org/10.1016/j. jmii.2014.04.004

4. Carrillo-Esper R, Peña-Pérez CA, Neri-Maldonado R, FloresRivera OI, de la Torre-León T, Pérez-Calatayud A, MezaMárquez JM, Alonso-Martínez D. Sepsis grave secundaria a bacteriemia por Chryseobacterium indologenes. Primer caso reportado en México. Med Int Méx. 2017;31(5): 633-36.

5. Hsueh PR, Teng LJ, Ho SW, Hsieh WC, Luh KT. Clincal and Microbiological Characteristics of Flavobacterium indologenes Infections Associted with Indwelling Devices. J Clin Microbiol. 1996;34(8):1908-13.

6. Robledo JA, López J, Sierra P, Robledo C, Pfaller MA, Jones RN. El programa de vigilancia antimicrobiana SENTRY en Colombia: hallazgos iniciales en tres hospitales de Medellín. Asociación Colombiana de Infectología.1999;3(2):100-7.

7. Gales A, Jones R, Andrade S, Sader H. Antimicrobial susceptibility patterns of unusual nonfermentative gram-negative bacilli isolated from Latin America: report from the SENTRY Antimicrobial Surveillance Program (1997-2002). Mem Inst Oswaldo Cruz. 2005;100(6):571-57. http://dx.doi. org/10.1590/S0074-02762005000600011

8. Kirby J, Sader H, Walsh T, Jones R. Antimicrobial susceptibility and epidemiology of a worldwide collection of Chryseobacterium spp: Report from the SENTRY Antimicrobial Surveillance Program (1997-2001). J Clin Microbiol. 2004;42(1):445-48.

9. Cimmino T, Rolain JM. Whole genome sequencing for deciphering the resistome of Chryseobacterium indologenes, an emerging multidrug-resistant bacterium isolated from a cystic fibrosis patient in Marseille, France. New Microbes New Infections. 2016;12:35-42. doi: 10.1016/j.nmni.2016.03.006

10. Aydin Teke T, Oz F, Metin O, Bayhan G, Gayretli Aydin Z, Oguz $M$, et al. Chryseobacterium indologenes. Septicemia in an Infant. Case Reports in Infectious Diseases. 2014;2014:1-4. http://dx.doi.org/10.1155/2014/270521

11. Lin $Y$, et al. Clinical and Microbiological Characteristics of Chryseobacterium indologenes Bacteremia. J Microbiol Immunol Infec. 2010;43(6):498-505. https://doi.org/10.1016/ S1684-1182(10)60077-1

12. Corbella M, Brandolini M, Cambieri P, Decembrino N, Pagani M, Bottazzi A, Muzzi A, Zecca M, Mariani B, Marone P. A catheter-related bloodstream infection caused by Chryseobacterium indologenes successfully treated with antibiotic-lock rescue therapy. New Microbiologica 2017; 4(40): 223-25.

13. Hsueh P, Hsiue T, Wu J, Teng L, Ho S, Hsieh W, et al. Flavobacterium indologenes Bacteremia: Clinical and Mi- 
crobiological Characteristics. Clinical Infectious Diseases. 1996;23(3):550-55.

14. Ozcan N, Dal T, Tekin A,Kelekci S, Can S, Ezin O, Kandemir I, Gul K.. Is Chryseobacterium indologenes a shunt-lover bacterium? A case report and review of the literature. Le Infezioni in Medicina. 2013; (4):312-16.

15. Aykac K, et al. Six cases during 2012-2015 and literature review of Chryseobacterium indologenes infections in pediatric patients. Canadian Journal of Microbiology. 2016;62(10):81219. https://doi.org/10.1139/cjm-2015-0800

16. Kodama Y, Nishimura M, Nakashima K. Central intravenous catheter-related bacteremia due to Cryseobacterium indologenes after cord blood transplantation. Rinsho Ketsueki. 2013;54:305-310.

17. Jackson MA, et al. The Use of Systemic and Topical Fluoroquinolones. Pediatrics. 2016;138(5). doi: 10.1542/ peds.2016-2706 